\title{
Quantitative Flow Cytometry Immunophenotypic Data in Myelodysplastic Syndromes (MDS)
}

\author{
Ha Nishino ${ }^{1}$, April Ewton ${ }^{2,3}$, A. Euton ${ }^{2,3}$,Youli Zu ${ }^{2}$, Audrey Ponce de Leon ${ }^{2}$, Kelty Baker $^{4}$, \\ George Carrum $^{4,5}$, Subhendu Chakraborty ${ }^{2}$, Lawrence Rice ${ }^{4}$, and Chung-Che Chang ${ }^{*, 2,3}$ \\ ${ }^{I}$ Department of Pathology, Baylor College of Medicine, Houston, TX 77030, USA \\ ${ }^{2}$ Department of Pathology, The Methodist Hospital and the Methodist Hospital Research Institute, Houston, TX 77030 , \\ USA \\ ${ }^{3}$ Department of Pathology, Weill Medical College of Cornell University, NY 10021, USA \\ ${ }^{4}$ Department of Medicine, The Methodist Hospital, Houston, TX 77030, USA \\ ${ }^{5}$ Department of Medicine, Baylor College of Medicine, TX 77030, USA
}

\begin{abstract}
Background: Recent studies using qualitative analysis of flow cytometry data have demonstrated various immunophenotypic abnormalities associated with myelodysplastic syndromes (MDS). However, there are limited reports assessing the ability of quantitative immunophenotypic analysis to discriminate MDS from other cytopenic conditions.

Design: Using flow cytometry, we studied 37 bone marrow specimens from 23 patients with MDS and 14 cytopenic patients with non-clonal hematologic disorders. Samples were analyzed quantitatively for percentages of T-cells, B-cells, NK cells, granulocytes, monocytes, blasts, erythroid precursors, and plasma cells; CD4:CD8 ratio; \% granulocyte subsets; $\%$ CD56+ monocytes; and \% erythroid precursor subsets.

Results: Quantitative analysis of immunophenotypic data in MDS patients compared to controls showed decreases in total granulocytes $(p=0.037)$ and more mature subsets of $\mathrm{CD} 11 \mathrm{~b}^{+} \mathrm{CD} 16^{\text {bright }}$ granulocytes $(p=0.0046)$ and $\mathrm{CD} 10^{+}$granulocytes $(p=0.022)$. MDS patients also showed a trending increase in subset percentage of CD56+monocytes $(p=0.056)$. Using receiver operating characteristic (ROC) analysis, cut-off values for these parameters favoring a diagnosis of MDS were identified as follows: total granulocytes $<60 \%, \mathrm{CD} 11 \mathrm{~b}^{+} \mathrm{CD} 16^{\text {bright }}$ granulocytic subset $<40 \%$, CD $10^{+}$granulocytic subset $<40 \%$, and $\mathrm{CD} 56^{+}$monocytic subset $>10 \%$. Subsequently, a scoring system was proposed whereby a score of one was assigned for the presence of each quantitative abnormality. Using this system on the original study population, the presence of at least two abnormalities (score $\geq 2$ ) revealed optimal sensitivity $(69.6 \%)$ and specificity (71.4\%) for a diagnosis of MDS.

Conclusion: These findings suggest that quantitative analysis of immunophenotypic data can be complementary to qualitative interpretation. These data may be useful for distinguishing MDS from non-clonal cytopenic disorders and warrant prospective study in additional MDS patients.
\end{abstract}

\section{INTRODUCTION}

Myelodysplastic syndromes (MDS) represent a heterogeneous group of clonal disorders of hematopoietic stem cells which are characterized by ineffective hematopoiesis, peripheral cytopenias, and multilineage dysplasia. The heterogeneity in the clinical, morphologic, biologic, and genetic characteristics of MDS has contributed to the complexity of this diagnosis. While it remains an inadequately characterized disorder, our understanding and management of MDS continues to be improved by our growing knowledge of the diagnostic and prognostic features relevant to its disease pathogenesis. Presently, the diagnosis of MDS is based on a combination of clinical history, peripheral blood/bone marrow morphology, and cytogenetics. Additional useful studies

*Address correspondence to this author at the Department of Pathology, The Methodist Hospital and the Methodist Hospital Research Institute, Houston, TX 77030, USA; E-mail: jeffchang@pol.net include immunophenotyping, in vitro colony growth assays, and molecular genetic testing. However, achieving minimal diagnostic criteria may represent challenges because morphologic evaluation can be hindered by nonspecific findings (e.g. marrow changes due to growth factor therapy, myelotoxic drugs, viral infection, alcohol, nutritional deficiency, and hypersplenism), marrow fibrosis or hypocellularity and the inconsistent presence of cytogenetic abnormalities (limited to $40-50 \%$ of cases) [1]. These limitations in our present diagnostic approach suggest the need for alternative strategies for evaluating MDS.

Flow cytometric immunophenotyping has recently emerged as a promising diagnostic method for characterizing MDS. Because normal development of hematopoietic cells is characterized by characteristic expression of specific antigens along expected stages of maturation, aberration from the predictable patterns of antigenic expression often represent a derangement in this process seen in various hema- 
tologic disorders and malignancies. Characterization of these immunophenotypic abnormalities by flow cytometry can provide important surrogate markers for disease.

The use of flow cytometry in the immunophenotypic analysis of hematologic malignancies has been an important practical application of this tool in the clinical setting. In current practice, flow cytometric immunophenotyping has shown clinical utility in the diagnosis, classification, prognostic stratification, and/or detection of residual disease in various hematologic malignancies including acute leukemias and non-Hodgkin's lymphomas; [2-7] however, its potential application to the diagnostic setting of MDS has not been as well established.

There are limited reports assessing the potential for discrimination of MDS from other cytopenic etiologies through quantitative analysis of immunophenotypic data. In a small series of cases, we previously demonstrated that quantitative analysis of decreased level of expression of maturational marker CD10 in marrow granulocytes may be a suggestive finding in the assessment of patients with a suspected diagnosis of MDS [8]. The primary objective of this study is to evaluate whether quantitative analysis of immunophenotypic data obtained by flow cytometry would yield practical and more objective discrimination of MDS from other cytopenic etiologies.

\section{MATERIALS AND METHODS}

\section{Patients}

We undertook a retrospective analysis of patients evaluated for MDS or unexplained cytopenia(s) at The Methodist
Hospital (Houston, TX) or its affiliate institutions between November 2002 and March 2005. Thirty-seven patients with flow cytometric studies and adequate clinical follow up were identified for inclusion in this study. Bone marrow aspirates from the following patients were analyzed by flow cytometry: 23 previously-untreated patients with confirmed MDS diagnosis by morphologic, clinical, and/or cytogenetic findings (7 RA [Refractory Anemia], 4 RARS [Refractory Anemia with Ringed Sideroblasts], 4 RCMD [Refractory Cytopenia with Multilineage Dysplasia], 3 RAEB-1 [Refractory Anemia with Excess Blasts-1], 3 RAEB-2 [Refractory Anemia with Excess Blasts-2], 1 5q- [Deletion 5q], and 1 MDSNOS [MDS-Not Otherwise Specified]) and 14 cytopenic patients with subsequent non-clonal hematologic disorders receiving marrow evaluation. Non-MDS patients are characterized by cytopenias of other etiologies in the absence of morphologic or cytogenetic findings for MDS. The diagnosis of MDS was established based on WHO (World Health Organization) classification and derived from combined clinical, morphologic, and cytogenetic data.

\section{Morphologic and Cytogenetic Evaluation}

Morphologic review of bone marrow aspirates, clot sections, and core biopsies was performed by one or more of hematologists (KB, LR, GC) or hematopathologists (EW, YZ, CC) who was blinded to flow cytometric findings. A minimum of 200 to 500 mononuclear cells per specimen was examined. The diagnosis and classification of MDS were based on WHO guidelines (Table 1) [9].

Cytogenetic data were acquired from bone marrow aspirates obtained concurrently with specimens analyzed by flow

Table 1. The World Health Organization (WHO) Classification and Criteria for MDS*

\begin{tabular}{|c|c|c|}
\hline Refractory anemia (RA) & $\begin{array}{c}\text { Anemia } \\
\text { Absent/rare blasts }\end{array}$ & $\begin{array}{l}\text { Isolated erythroid dysplasia } \\
<5 \% \text { blasts } \\
<15 \% \text { ringed sideroblasts }\end{array}$ \\
\hline Refractory anemia with ringed sideroblasts (RARS) & $\begin{array}{c}\text { Anemia } \\
\text { Absent blasts }\end{array}$ & $\begin{array}{l}\text { Isolated erythroid dysplasia } \\
\quad<5 \% \text { blasts } \\
\geq 15 \% \text { ringed sideroblasts }\end{array}$ \\
\hline Refractory cytopenia with multilineage dysplasia (RCMD) & $\begin{array}{l}\text { Cytopenias ( } \geq 2 \text { lines }) \\
\text { Absent/rare blasts } \\
\text { Absent Auer rods } \\
\text { Monocytes }<1,000 / \mu \mathrm{L}\end{array}$ & $\begin{array}{c}\geq 10 \% \text { dysplasia in } \geq 2 \text { myeloid cell lines } \\
<5 \% \text { blasts } \\
\text { Absent Auer rods } \\
<15 \% \text { ringed sideroblasts }\end{array}$ \\
\hline Refractory anemia with excess blasts - 1 (RAEB-1) & $\begin{array}{c}\text { Cytopenias } \\
<5 \% \text { blasts } \\
\text { Absent Auer rods } \\
\text { Monocytes }<1,000 / \mu \mathrm{L}\end{array}$ & $\begin{array}{c}\text { Unilineage or multilineage dysplasia } \\
5-9 \% \text { blasts } \\
\text { Absent Auer rods }\end{array}$ \\
\hline Refractory anemia with excess blasts - 2 (RAEB-2) & $\begin{array}{c}\text { Cytopenias } \\
5-19 \% \text { blasts } \\
\pm \text { Auer rods } \\
\text { Monocytes }<1,000 / \mu \mathrm{L}\end{array}$ & $\begin{array}{c}\text { Unilineage or multilineage dysplasia } \\
10-19 \% \text { blasts } \\
\pm \text { Auer rods }\end{array}$ \\
\hline
\end{tabular}


cytometry. Karyotyping studies were performed on 20 metaphase nuclei using standard G-banding with trypsin-Giemsa staining (DynaGene Laboratory, Houston, TX).

\section{Flow Cytometry}

In preparation for flow cytometric studies, heparinized bone marrow aspirates were stained within six hours of specimen collection and processed for surface marker analysis. After dilution in phosphate-buffered saline with $1 \%$ FCS (Cambrex Bio Science, Walkersville, MD) to an approximate concentration of $10 \times 10^{6}$ cells $/ \mathrm{mL}$ followed by filtration, specimens were processed using a whole blood lysis technique (Becton Dickinson, San Jose, CA). Briefly, 100 $\mu 1$ $\left(1 \times 10^{6}\right.$ cells $)$ of diluted bone marrow cell suspension were incubated with directly conjugated monoclonal antibodies (Becton Dickinson Immunocytometry Systems Inc., San Jose, CA) for 15 minutes at room temperature (Table 2). Erythrocyte lysis was achieved by adding $2 \mathrm{ml}$ ammonium chloride lysing solution to each fluorochrome tube and incubating for an additional 10 minutes. Finally, leukocytes were washed twice and then resuspended in $0.5 \%$ paraformaldehyde prior to flow cytometric analysis.

Table 2. Panel of Monoclonal Antibodies

\begin{tabular}{|c|}
\hline Monoclonal Antibodies \\
\hline \hline FITC/PE/PerCP/APC \\
\hline $\mathrm{CD} 22 / \mathrm{CD} 11 \mathrm{c} / \mathrm{CD} 45 / \mathrm{CD} 14$ \\
\hline $\mathrm{CD} 2 / \mathrm{CD} 56 / \mathrm{CD} 3 / \mathrm{CD} 45 \mathrm{RO}$ \\
\hline $\mathrm{CD} 7 / \mathrm{CD} 4 / \mathrm{CD} 3 / \mathrm{CD} 8$ \\
\hline $\mathrm{CD} 20 / \mathrm{CD} 10 / \mathrm{CD} 19 / \mathrm{CD} 38$ \\
\hline $\mathrm{CD} 36 / \mathrm{CD} 64 / \mathrm{CD} 45 / \mathrm{CD} 34$ \\
\hline $\mathrm{CD} 16 / \mathrm{CD} 13 / \mathrm{CD} 45 / \mathrm{CD} 11 \mathrm{~b}$ \\
\hline $\mathrm{CD} 45 / \mathrm{CD} 33 / \mathrm{HLA}-\mathrm{DR} / \mathrm{CD} 34$ \\
\hline $\mathrm{CD} 71 / \mathrm{GPA} / \mathrm{CD} 45 / \mathrm{CD} 117$ \\
\hline FITC: fluorescein isothiocyanate; PE: phycoerythrin; PerCP: peridinin chlorophyll
\end{tabular}
protein; APC: allophycocyanin; GPA: glycophorin A.

Six-parameter, four-color flow cytometry was performed using a FACSCalibur flow cytometer (Becton Dickinson) equipped with $15-\mathrm{mW}$ Argon (488 nm) and $10-\mathrm{mW} \mathrm{HeNe}$ $(633 \mathrm{~nm})$ lasers. The percentage of positive cells for each marker was determined using recommended manufacturer configurations. Autocompensation using Calibrite beads (Becton Dickinson) was used to standardize instrument electronics. Background fluorescence levels for each specimen were established using cells incubated with appropriate fluorochrome-coupled isotype control antibodies. Fluorochrome compensation was adjusted electronically using normal control peripheral blood leukocytes labeled with fluorescein isothiocyanate (FITC)-coupled anti-CD7, peridinin chlorophyll protein (PerCP)-coupled anti-CD3, allophycocyanin (APC)-coupled anti-CD8, and phycoerythrin (PE)coupled anti-CD4. Corresponding isotype negative controls were included in all studies.

10,000 to 15,000 total events were acquired from each tube with forward and side scatter signals. Data were collected and processed using CellQuest software (Becton Dickinson, San Jose, CA). Flow cytometric data were analyzed using Paint-a-Gate software (Becton Dickinson). Immunophenotypic patterns of subsets of mononuclear cells were determined through cluster analysis of data according to cell size (approximated by forward angle light scatter properties), cytoplasmic granularity (approximated by side angle light scatter properties), and antigen expression patterns.

\section{Statistical Analysis}

Statistical analyses were performed using StatView Version 5.0.1 software (SAS Institute Inc., Cary, NC). The percentage of individual subsets expressing specific surface markers among MDS patients was each compared with control patients. The statistical significance of differences observed between groups was measured using the Student $t$-test or Mann-Whitney U test. Kolmogorov-Smirnov's test for normality and Levene's test for equality of variances were used in evaluating sample distribution and qualifying parametric data. The Mann-Whitney $U$ test for nonparametric analysis was used in cases where normality assumptions were not satisfied. Receiver operating characteristic (ROC) analyses were performed using JROCFIT 1.0.2 and JLABROC4 1.0.1 (John Eng, Johns Hopkins University, Baltimore, Maryland).

\section{RESULTS}

Characteristics of patient groups are summarized as follows. The mean age at diagnosis for MDS patients $(n=23)$ was $73.8 \pm 2.2$ years with a range of 47-87. For control patients $(n=14)$, the mean age was $69.4 \pm 4.6$ years with a range of 42-83. There were no significant differences in age or gender between these groups.

Samples were analyzed qualitatively and quantitatively for percentages of T-cells $\left(\mathrm{CD}^{+}\right)$, B-cells $\left(\mathrm{CD} 20^{+}\right)$, NK cells (CD3-CD56+), granulocytes (moderate CD45/high side scatter), monocytes $\left(\mathrm{CD} 14^{+} \mathrm{CD} 11 \mathrm{c}^{+}\right)$, blasts ( $\operatorname{dim} \mathrm{CD} 45 /$ low side scatter, $\mathrm{CD} 34^{+}$and/or $\mathrm{CD} 117^{+}$), erythroid precursors $\left(\mathrm{CD} 71^{+} \mathrm{CD} 45-\right)$, and plasma cells (bright $\left.\mathrm{CD} 38\right)$; $\mathrm{CD} 4: \mathrm{CD} 8$ ratio; percentage of granulocyte subsets $\left(\mathrm{CD}^{+} 0^{+}, \mathrm{CD} 10-\right.$, CD $36^{+} \mathrm{CD}_{4}{ }^{+}, \quad \mathrm{CD} 36-\mathrm{CD} 64^{+}, \quad \mathrm{CD} 11 \mathrm{~b}-\mathrm{CD} 16-, \quad \mathrm{CD} 11 \mathrm{~b}^{+}$ CD16- or $\mathrm{CD} 11 \mathrm{~b}^{+} \mathrm{CD} 16^{\text {bright }}$ granulocytes per total granulocytes); percentage of CD56+ monocytes; and percentage of erythroid precursor subsets (glycophorin A+ or A- erythroid precursors per total erythroid cells). As in previous studies, qualitative analysis of these data showed abnormal patterns of expression in myelomonocytic lineages and/or erythroid lineage for the vast majority of MDS patients (22/23) and in a small proportion $(2 / 14)$ of non-MDS cytopenia patients. Further analysis of these qualitative findings is not reported here since these findings have been described in detail in several recent studies [12-14, 16].

Quantitative analysis of flow cytometric data in MDS patients compared to non-MDS controls showed decreased total granulocytes (Fig. (1A), $62.2 \pm 19.7 \%$ vs $74.7 \pm 11.3 \%$, $p=0.047$, Mann-Whitney U test) and significantly decreased subset percentages of more mature granulocyte subsets: CD11b+CD16 ${ }^{\text {bright }} /$ granulocytes (Fig. (1B), $33.3 \pm 15.8 v s$ $49.1 \pm 14.7, p=0.0046$, Student $t$-test) and CD10+/granulo- 


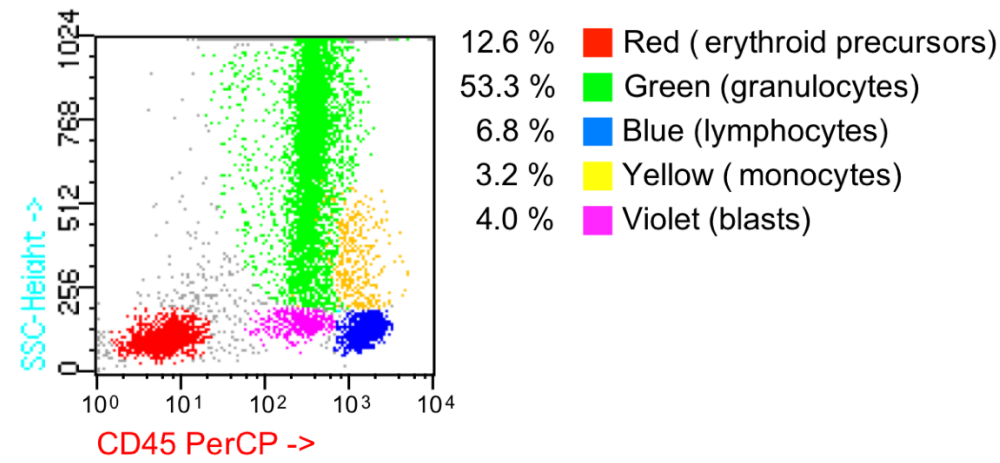

1A. \% total granulocytes = moderate CD45+ cells with high side scatter (green) [MDS Patient \#20]. The yellow cell population represents monocytes; the violet cell population represents blasts; the blue cell population represents lymphocytes; the red cell population represents erythroid precursors.

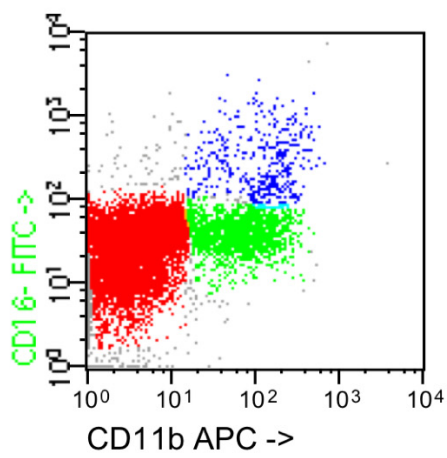
$38.1 \%$ Red (CD11b-CD16- granulocytes)
$11.5 \%$ Green (CD11b+CD16 $6^{\text {weak }}$ granulocytes)
$3.7 \%$ Blue (CD11b+CD16 bright granulocytes)

CD116 APC ->

1B. \% CD11b+CD16 bright granulocytes $=\mathrm{CD} 11 \mathrm{~b}+\mathrm{CD} 16^{\text {bright }}$ (blue, in \% of total cells) $/\left[\mathrm{CD} 11 \mathrm{~b}+\mathrm{CD} 16^{\text {bright }}\right.$ (blue) + CD11b+CD16 ${ }^{\text {weak }}$ (green) + CD11b-CD16- (red), in \% of total cells] $=3.7 /(3.7+11.5+38.1)$ for the example shown [MDS Patient \#20].

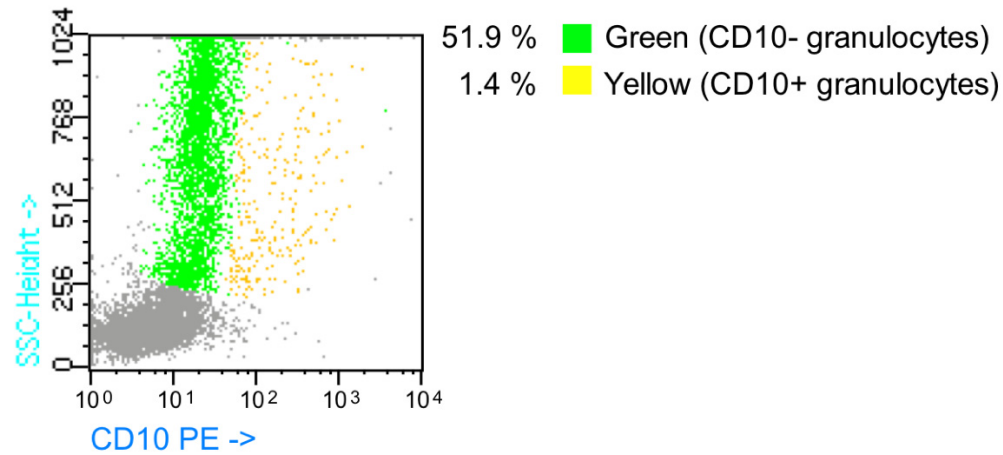

1C. $\% \mathrm{CD} 10+$ granulocytes $=\mathrm{CD} 10+$ granulocytes (yellow, in \% of total cells) $/$ total granulocytes (yellow + green, in $\%$ of total cells $)=1.4 /(1.4+51.9)$ for the example shown [MDS Patient \#20]. The gray cell population represents non-granulocytes.

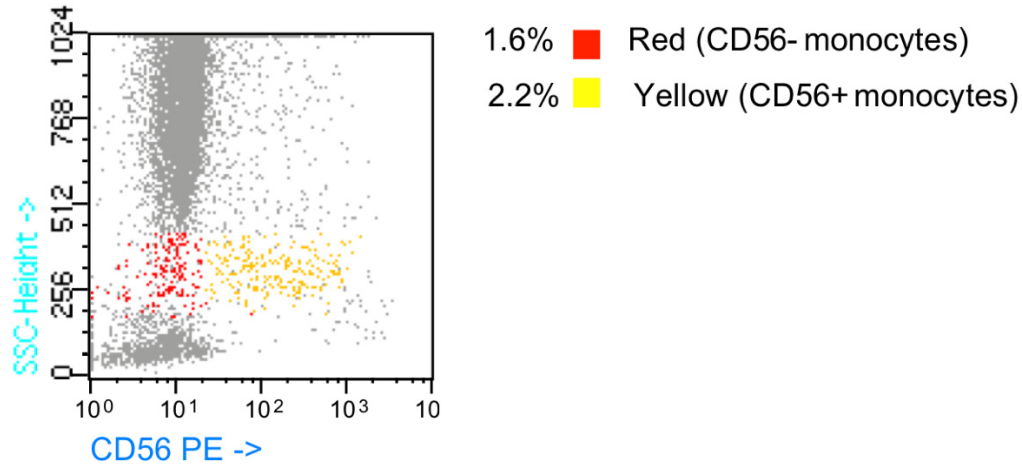

1D. $\%$ CD56+ monocytes $=$ CD56 + monocytes (yellow, in $\%$ of total cells) $/$ total monocytes (yellow + red, in $\%$ of total cells $)=$ $2.2 /(1.6+2.2)$ for the example shown [MDS Patient \#10].

Fig. (1). Quantitative analysis of flow cytometric data in MDS patients compared to non-MDS controls. 
Table 3. Characteristics of MDS Patients and Selected Immunophenotypic Data

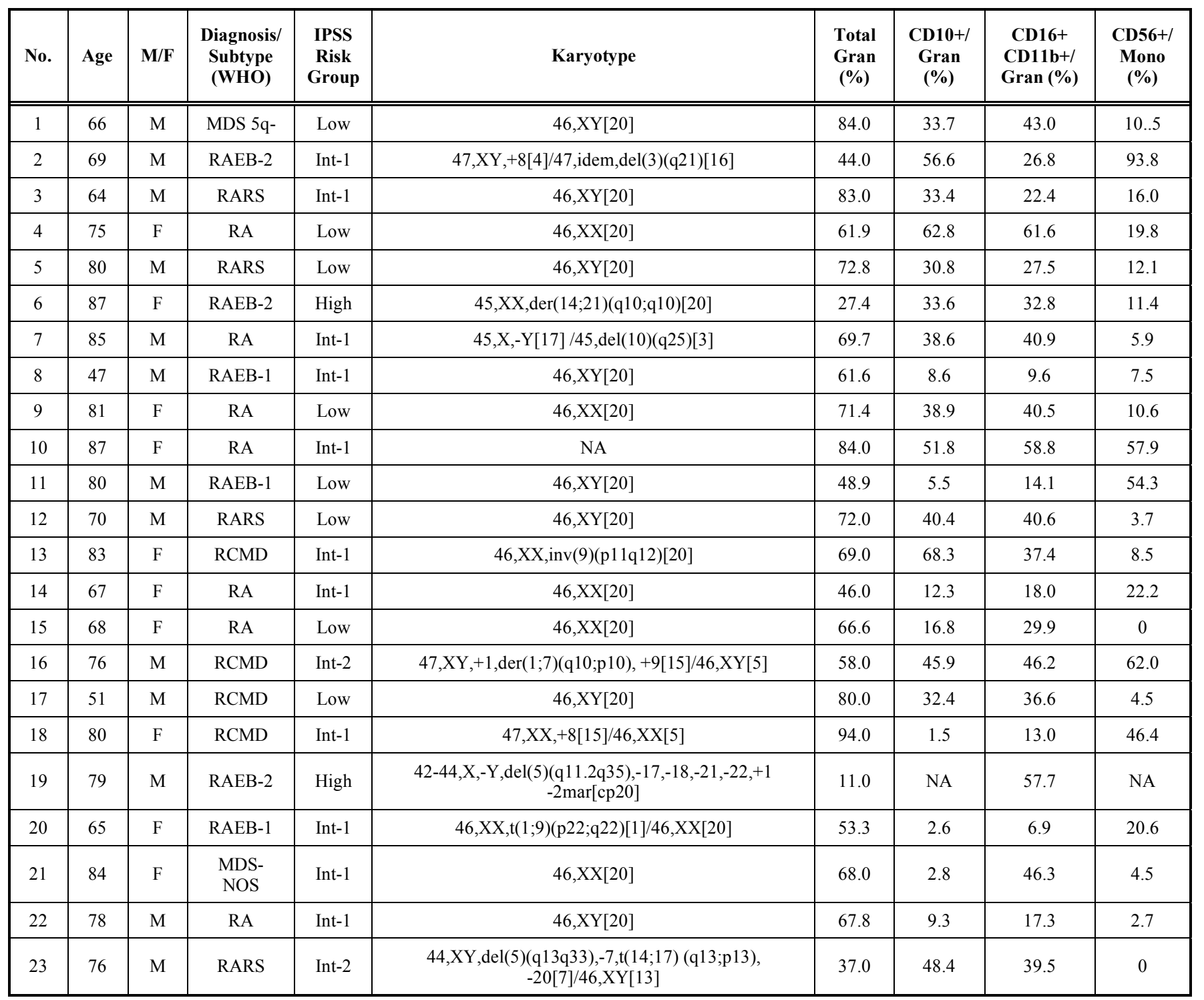

Gran: total granulocytes; Mono: total monocytes; Int: intermediate; NA: not available.

cytes (Fig. (1C), 30.7 \pm 20.5 vs $46.1 \pm 11.0, p=0.022$, MannWhitney U test), (Tables 3, 4 and 5). The MDS cohort demonstrated corresponding increases in percentages of less mature granulocytic subsets (i.e. CD11b-/CD16 ${ }^{\text {weak }}$ or CD10-). MDS patients also showed a trending increase in the percentage of monocytic subset expressing CD56 (Fig. (1D), CD56 $6^{+}$monocytes $)$compared to controls $(21.5 \pm 24.9$ vs 6.9 $\pm 3.9, p=0.056$, Mann-Whitney $\mathrm{U}$ test), (Tables 3, 4 and 5). There were no significant differences quantitatively in other cellular populations identified between the MDS and non-MDS patients including the B-cell and T-cell subsets (data not shown). Additionally, there were no significant differences in percentage of blasts between these groups of patients; this observation may be partly due to the limited number of high-grade MDS cases (3 RAEB-I, 3 RAEB-II) in our cohort of MDS patients.

To further identify more objective criteria helpful in establishing a diagnosis of MDS, numerical thresholds for abnormal expression of these antigenic phenotypes were established based on receiver operating characteristic (ROC) analysis (Table 6) for the optimal sensitivity and specificity for a diagnosis of MDS. The proposed critical (cut-off) values for these significant immunophenotypic parameters were then identified as follows: percentage of total granulocytes $<$ $60 \%$, percentage of $\mathrm{CD} 11 \mathrm{~b}^{+} \mathrm{CD} 16^{\text {bright }}$ granulocytic subset $<$ $40 \%$, percentage of $\mathrm{CD} 10^{+}$granulocytic subset $<40 \%$, and percentage of $\mathrm{CD}^{2} 6^{+}$monocytic subset $>10 \%$. The combination of these parameters were then used to establish a scoring system with score 1 for the presence and 0 for the absence of any quantitative abnormalities. Using this scoring system, the presence of two of these characterized immunophenotypes (score 2) was determined to have optimal sensitivity $(69.6 \%)$ and specificity $(71.4 \%)$ for a diagnosis of MDS.

\section{DISCUSSION}

In the past decade, several studies have reported distinct immunophenotypic characteristics associated with MDS (e.g. asynchronous maturational antigen expression, aberrant expression of lineage-specific markers, and abnormal levels of expression of complement receptors, leukocyte activation 
Table 4. Characteristics of Non-MDS Patients and Selected Immunophenotypic Data

\begin{tabular}{|c|c|c|c|c|c|c|c|c|}
\hline No & Age & $\mathbf{M} / \mathbf{F}$ & Diagnosis & Karyotype & $\begin{array}{l}\text { Total Gran } \\
\text { (\%) }\end{array}$ & $\begin{array}{c}\text { CD10+/Gran } \\
(\%)\end{array}$ & 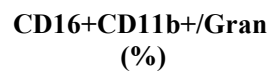 & $\begin{array}{c}\text { CD56+/ Mono } \\
\text { (\%) }\end{array}$ \\
\hline 1 & 57 & $\mathrm{~F}$ & N/A & $46, \mathrm{XX}[20]$ & 52.0 & 51.3 & 51.9 & 13.9 \\
\hline 2 & 36 & M & HIV infection & $46, \mathrm{XY}[20]$ & 80.0 & 42.0 & 50.4 & 0 \\
\hline 3 & 77 & M & ACD & $45, \mathrm{X},-\mathrm{Y}[3] / 46, \mathrm{XY}[17]$ & 68.0 & 60.3 & 67.8 & 10.0 \\
\hline 4 & 69 & M & ACD & $46, X Y[20]$ & 78.0 & 36.9 & 42.3 & 9.2 \\
\hline 5 & 83 & M & IDA & $45, \mathrm{X},-\mathrm{Y}[4] / 46, \mathrm{XY}[16]$ & 78.0 & 42.3 & 39.0 & 10.3 \\
\hline 6 & 81 & $\mathrm{~F}$ & CLL & NA & 89.0 & 35.2 & 34.8 & 10.0 \\
\hline 7 & 74 & $\mathrm{~F}$ & ITP & $46, \mathrm{XX}[20]$ & 86.8 & 66.9 & 67.9 & 7.0 \\
\hline 8 & 77 & $\mathrm{~F}$ & $\mathrm{HL}$ & $46, \mathrm{XX}[20]$ & 60.0 & 33.2 & 36.7 & 5.4 \\
\hline 9 & 42 & M & ESRD & $46, \mathrm{XY}[20]$ & 69.0 & 29.7 & 34.8 & 3.1 \\
\hline 10 & 75 & M & Sarcoidosis & $46, X Y[20]$ & 80.6 & 48.4 & 49.6 & 2.4 \\
\hline 11 & 77 & M & ITP & $46, \mathrm{XY}[20]$ & 80.0 & 51.1 & 44.5 & 9.7 \\
\hline 12 & 80 & $\mathrm{M}$ & ACD & $46, \mathrm{XY}[20]$ & 72.0 & 48.5 & 45.4 & 6.3 \\
\hline 13 & 72 & $\mathrm{~F}$ & Tuberculosis & $46, \mathrm{XY}[20]$ & 63.0 & 41.7 & 37.9 & 3.3 \\
\hline 14 & 71 & $\mathrm{~F}$ & ACD & NA & 90.0 & 59.8 & 83.8 & 8.5 \\
\hline
\end{tabular}

Gran: total granulocytes; HIV: Human Immunodeficiency Virus; Mono: total monocytes; ACD: Anemia of Chronic Disease; N/A: not available; IDA: Iron Deficiency Anemia; CLL: Chronic Lymphocytic Leukemia; ITP: Idiopathic Thrombocytopenic Purpura; HL: Hodgkin Lymphoma; ESRD: End-Stage Renal Disease.

antigens, adhesion molecules, apoptosis-related antigens, hematopoietic stem cell antigens, cytokine receptors, and lymphoid surface marker expression) that suggest the possible role of flow cytometric immunophenotyping in supporting this diagnosis [10-16]. These studies have demonstrated recurrent immunophenotypic abnormalities in MDS distinguishing this hematologic disorder from normal patients. These reported abnormalities of myeloid lineage include decreased granularity, decreased CD45 expression, decreased CD10 expression, absent CD13 expression, absent CD33 expression, aberrant CD56 expression, aberrant CD34 expression, aberrant expression of nonmyeloid antigens, abnormal relationship of CD11b/HLA-DR, abnormal relationship of CD11b/CD16, and abnormal relationship of CD13/CD16 [8, 10, 12, 13, 16-19]. Abnormalities of the erythroid lineage include decreased CD71 expression, abnormal relationship of CD45/CD71, abnormal relationship of CD45/Glycophorin A, and abnormal relationship of CD71/Glycophorin A [14, 16, 20]. Limited changes in the megakaryocytic lineage have been reported due to the infrequent presence of this cellular component in bone marrow and corresponding absence of appropriate antibodies for detection by flow cytometry.

In our study, quantitative analysis of these immunophenotypic data is considered in an attempt to identify more objective criteria that may be useful in the evaluation of patients receiving bone marrow evaluation for unexplained cytopenias. Surface antigens previously reported to have abnormal expression in MDS are evaluated in this study and consist of markers for myelomonocytic and erythroid maturation. The flow cytometry instrument used in this study features the four-color technology commonly used in clinical practice. With four-color flow cytometry, our evaluation of these particular markers is limited to four antibody combina- tions; therefore, relevant and practical combinations are exploited to maximize the acquisition of information. While the addition of useful antibodies including CD45 would allow for more accurate gating, the cost associated with more advanced six- to eight-color flow cytometry would not be practical in the clinical setting. However, this more advanced technology enabling concurrent evaluation of additional pertinent antibodies may be useful in the prospective validation of data obtained from the standard technology. Using fourcolor flow cytometry, our results indicate that percentages of

Table 5. Quantitative Immunophenotypic Differences in Ranulocytic and Monocytic Lineages Between MDS and Control Patients

\begin{tabular}{|c|c|c|c|}
\hline Subset & MDS $(\mathbf{n}=\mathbf{2 3}) *$ & Control $(\mathbf{n}=\mathbf{1 4}) *$ & $\boldsymbol{p}$ \\
\hline \hline Total Granulocytes & $62.2 \pm 19.7$ & $74.7 \pm 11.3$ & $<0.05^{\dagger}$ \\
\hline CD16+CD11b+/Gran & $33.3 \pm 15.8$ & $49.1 \pm 14.7$ & 0.010 \\
\hline CD10+/Gran & $30.7 \pm 20.5 \cdot$ & $46.1 \pm 11.0$ & $0.022^{\dagger}$ \\
\hline CD56+/Mono & $21.5 \pm 24.9 \cdot$ & $6.9 \pm 3.9$ & $0.056^{\dagger}$ \\
\hline
\end{tabular}

*Mean \pm standard deviation.

$\cdot \mathrm{n}=22$ patients.

${ }^{\dagger}$ Mann-Whitney U test

Student $t$-test.

Gran: total granulocytes; Mono: total monocytes.

total granulocytes, $\mathrm{CD} 11 \mathrm{~b}^{+} / \mathrm{CD} 16^{\text {bright }}$ granulocyte subset, $\mathrm{CD} 0^{+}$granulocyte subset were significantly lower in MDS patients than the non-MDS patients, and that $\mathrm{CD}_{5} 6^{+}$monocyte subset trended to increase in MDS patients. Three of these immunophenotypic characteristics $\left(\mathrm{CD} 11 \mathrm{~b}^{+} / \mathrm{CD} 16^{\text {bright }}\right.$ granulocytic expression, $\mathrm{CD} 10^{+}$neutrophilic expression, and 

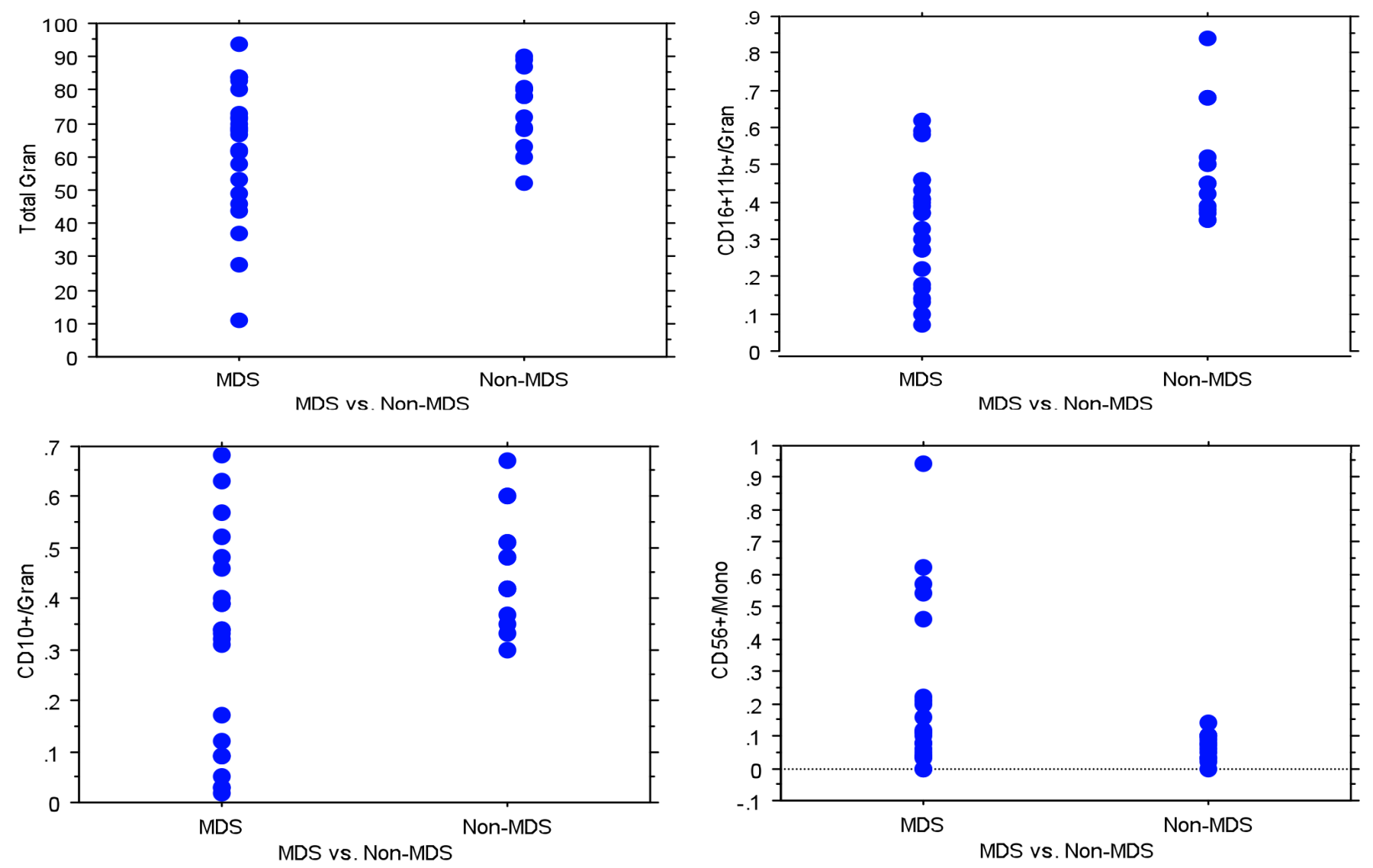

Fig. (2). Bivariate scattergrams illustrating immunophenotypic differences in granulocytic and monocytic lineages between MDS and control patients

$\mathrm{CD}^{+} 6^{+}$monocytic expression) are consistent with antigenic abnormalities previously described in MDS using qualitative interpretation $[8,16,20]$.

Table 6. Sensitivity and Specificity for Diagnosing MDS Using Immunophenotypic Abnormality Scores

\begin{tabular}{|c|c|c|}
\hline \multirow{2}{*}{$\begin{array}{l}\text { Immunophenotypic Abnormality } \\
\text { Scores* }\end{array}$} & \multicolumn{2}{|c|}{ MDS } \\
\hline & Sensitivity & Specificity \\
\hline$\geq 1$ & $95.6 \%$ & $50.0 \%$ \\
\hline$\geq 2$ & $69.6 \%$ & $71.4 \%$ \\
\hline$\geq 3$ & 39.1 & $100 \%$ \\
\hline$\geq 4$ & $21.1 \%$ & $100 \%$ \\
\hline \multicolumn{3}{|c|}{$\begin{array}{l}\text { Assigned score }=1 \text { for each of the following: } \\
\text { Total granulocyte }<60 \% \text { of total cells. } \\
\text { CD } 11 b^{+} \text {CD } 16^{\text {bight }} \text { granulocyte subset }<40 \% \text { of total granulocytes. } \\
\text { CD } 10^{+} \text {granulocyte subset }<40 \% \text { of total granulocytes. } \\
\text { CD } 56^{+} \text {monocyte subset }>10 \% \text { of total monocytes. } \\
\text { These cutoff values are determined using Receiver Operating Curve analysis. } \\
\text { Sensitivity = True Positives / (True Positives + False Negatives). } \\
\text { Specificity = True Negatives / (True Negatives + False Positives). }\end{array}$} \\
\hline
\end{tabular}

Using ROC analysis, we further identify thresholds demonstrating optimal discrimination between the MDS and non-MDS patients as percentage of total granulocytes < $60 \%$, percentage of $\mathrm{CD} 11 \mathrm{~b}^{+} \mathrm{CD} 16^{\text {bright }}$ granulocytic subset $<$ $40 \%$, percentage of $\mathrm{CD} 10^{+}$granulocytic subset $<40 \%$, and percentage of $\mathrm{CD}_{5} 6^{+}$monocytic subset $>10 \%$. Similar to qualitative analysis reported in the previous studies, the quantitative abnormalities are very sensitive for the MDS patients with only one patient not showing any abnormalities based on ROC curve assigned cut-off values; however, the specificity of these abnormalities is relatively low in the current study. As shown in Tables $\mathbf{4}$ and $\mathbf{5}$, significant proportions of non-MDS cytopenic patients also carry these abnormalities. This observation may be attributed to previouslyreported findings of low-level antigenic expression seen in clinical settings of growth factor therapy and regenerative bone marrow $[10,21]$. However, the mechanisms leading to such changes remain uncertain since our non-MDS controls are represented by patients demonstrating varying degrees of cytopenia of different etiologies (Table 4). It is possible that some of these patients may have been previously treated with growth factors, even though we did not identify such information in our chart review.

To further maximize the differentiating power between MDS and non-MDS, we evaluated the possibility of considering these four parameters in combination using a scoring system. Our results suggest that the presence of two abnormalities (score $\geq 2$ ) provides optimal sensitivity $(69.6 \%)$ and specificity $(71.4 \%)$ for a diagnosis of MDS. Of note, only MDS patients $(\mathrm{n}=9)$ demonstrate at least three immunophenotypic abnormalities (score $\geq 3$ ) suggesting that this cut-off is relatively specific despite limited sensitivity ( 9 out of 23 MDS patients) for the diagnosis of MDS. Although the significance of the sensitivity and specificity of these cutoff values is limited by the relatively small number of patients, these results suggest that discriminatory immunophenotypes can be quantified to develop more objective characterization 
of MDS. Furthermore, because non-MDS patients with other cytopenias are used as controls in this study, our results support the potential application of the quantitative approach in the evaluation of this subset of patients who frequently present for suspected MDS.

The prospective value of our quantitative approach can be illustrated in the example of the measurable difference in the detection of CD56 expression in monocytes between MDS and non-MDS patients. Abnormal expression of CD56 in monocytes previously described by Stetler-Stevenson and colleagues [16] is a characteristic pattern observed in our MDS cohort. However, limited aberrant CD56 expression in monocytes is also observed in the majority of non-MDS control patients (13/14) with lower quantified levels of expression when compared to MDS patients. This finding suggests that the quantitative consideration of the immunophenotypic data may contribute discriminatory value and be complementary to the qualitative pattern identification approach supported by the US-Canadian Consensus Recommendations on Immunophenotypic Analysis of Hematologic Neoplasia by Flow Cytometry [22, 23].

However, it should be noted that our quantitative approach in the use of flow cytometry has limitation in interlaboratorial correlation and validation. Variable sample processing methods, different instrument settings, and use of different antibodies in different laboratories are likely to change the data presented in this study although the similar abnormalities are expected. These are important considerations in the interpretation of results for individual laboratories. Laboratories may have to establish their own cut-off values using the methods outlined in the current study. Furthermore, the use of flow cytometry features inherent limitations for detection of intracellular markers that would require confirmation by immunohistochemistry. However, the immunophenotypic markers investigated in this study are all surface markers that are reliably detected by flow cytometry.

In summary, our findings suggest that quantitative interpretation of these immunophenotypic abnormalities can establish objective criteria that complement our current qualitative approach to evaluation of flow cytometric data. These characteristic immunophenotypic abnormalities offer additional data that can be correlated with morphologic, cytogenetic, and clinical findings to provide a valuable approach the evaluation and diagnosis of myelodysplastic syndromes. Future studies of larger sample sizes with inclusion of varied patient populations are warranted to confirm the significance of these observations.

\section{REFERENCES}

[1] Greenberg PL, Ed. Myelodysplastic syndrome. 3rd Ed. New York: Churchill Livingston; 2000.

[2] Sievers EL, Lange BJ, Alonzo TA, et al. Immunophenotypic evidence of leukemia after induction therapy predicts relapse: results from a prospective Children's Cancer Group study of 252 patients with acute myeloid leukemia. Blood 2003; 101:3398-406.
[3] Bene MC, Bernier M, Castoldi G, et al. Impact of immunophenotyping on management of acute leukemias. Haematologica 1999; 84: 1024-34.

[4] Wells DA, Hall MC, Shulman HM, Loken MR. Occult B cell malignancies can be detected by three-color flow cytometry in patients with cytopenias. Leukemia 1998; 12: 2015-23.

[5] Jennings CD, Foon KA. Flow cytometry: recent advances in diagnosis and monitoring of leukemia. Cancer Invest 1997; 15: 384-99.

[6] Davis BH, Foucar K, Szczarkowski W, et al. U.S.-Canadian Consensus recommendations on the immunophenotypic analysis of hematologic neoplasia by flow cytometry: medical indications. Cytometry 1997; 30: 249-63.

[7] Borowitz MJ, Guenther KL, Shults KE, Stelzer GT. Immunophenotyping of acute leukemia by flow cytometric analysis. Use of CD45 and right-angle light scatter to gate on leukemic blasts in three-color analysis. Am J Clin Pathol 1993; 100: 534-40.

[8] Chang CC, Cleveland RP. Decreased CD10-positive mature granulocytes in bone marrow from patients with myelodysplastic syndrome. Arch Pathol Lab Med 2000; 124: 1152-6.

[9] Vardiman JW, Harris NL, Brunning RD. The World Health Organization (WHO) classification of the myeloid neoplasms. [see comment]. Blood 2002; 100: 2292-302.

[10] Wells DA, Benesch M, Loken MR, et al. Myeloid and monocytic dyspoiesis as determined by flow cytometric scoring in myelodysplastic syndrome correlates with the IPSS and with outcome after hematopoietic stem cell transplantation. Blood 2003; 102: 394-403.

[11] Elghetany MT. Surface marker abnormalities in myelodysplastic syndromes. Haematologica 1998; 83: 1104-15.

[12] Benesch M, Deeg HJ, Wells D, Loken M. Flow cytometry for diagnosis and assessment of prognosis in patients with myelodysplastic syndromes. Hematology 2004; 9: 171-7.

[13] Del Canizo MC, Fernandez ME, Lopez A, et al. Immunophenotypic analysis of myelodysplastic syndromes. Haematologica 2003; 88: 402-7.

[14] Maynadie M, Picard F, Husson B, et al. Immunophenotypic clustering of myelodysplastic syndromes. Blood 2002; 100: 2349-56.

[15] Ogata K, Nakamura K, Yokose N, et al. Clinical significance of phenotypic features of blasts in patients with myelodysplastic syndrome. Blood 2002; 100: 3887-96.

[16] Stetler-Stevenson M, Arthur DC, Jabbour N, et al. Diagnostic utility of flow cytometric immunophenotyping in myelodysplastic syndrome. Blood 2001; 98: 979-87.

[17] Xu D, Schultz C, Akker Y, et al. Evidence for expression of early myeloid antigens in mature, non-blast myeloid cells in myelodysplasia. Am J Hematol 2003; 74: 9-16.

[18] Bowen KL, Davis BH. Abnormal patterns of expression of CD16 and $\mathrm{CD} 11 \mathrm{~b}$ antigens by developing neutrophils in the bone marrow of patients with myelodysplastic syndrome. Lab Hematol 1997: 292-298.

[19] Guyotat D, Campos L, Thomas X, et al. Myelodysplastic syndromes: a study of surface markers and in vitro growth patterns. Am J Hematol 1990; 34: 26-31.

[20] Malcovati L, Della Porta MG, Lunghi M, et al. Flow cytometry evaluation of erythroid and myeloid dysplasia in patients with myelodysplastic syndrome. Leukemia 2005; 19: 776-83.

[21] Smith WB, Guida L, Sun Q, et al. Neutrophils activated by granulocyte-macrophage colony-stimulating factor express receptors for interleukin-3 which mediate class II expression. Blood 1995; 86: 393844.

[22] Borowitz MJ, Bray R, Gascoyne R, et al. U.S.-Canadian Consensus recommendations on the immunophenotypic analysis of hematologic neoplasia by flow cytometry: data analysis and interpretation. Cytometry $1997 ; 30: 236-44$.

[23] Stelzer GT, Marti G, Hurley A, McCoy P, Jr., Lovett EJ, Schwartz A. U.S.-Canadian Consensus recommendations on the immunophenotypic analysis of hematologic neoplasia by flow cytometry: standardization and validation of laboratory procedures. Cytometry 1997; 30: $214-30$ 